\title{
Generalizations of Sherman's inequality by Lidstone's interpolating polynomial
}

Ravi P Agarwal' ${ }^{1}$, Slavica Ivelić Bradanović ${ }^{*}$ and Josip Pečarić ${ }^{3}$

"Correspondence: sivelic@gradst.hr

${ }^{2}$ Faculty of Civil Engineering,

Architecture and Geodesy,

University of Split, Matice hrvatske

15, Split, 21000, Croatia

Full list of author information is

available at the end of the article

\begin{abstract}
In majorization theory, the well-known majorization theorem plays a very important role. A more general result was obtained by Sherman. In this paper, concerning $2 n$-convex functions, we get generalizations of these results applying Lidstone's interpolating polynomials and the Čebyšev functional. Using the obtained results, we generate a new family of exponentially convex functions. The results are some new classes of two-parameter Cauchy type means.
\end{abstract}

MSC: 26D15

Keywords: majorization; n-convexity; Schur-convexity; Sherman's theorem; Lidstone interpolating polynomial; Čebyšev functional; Grüss type inequalities; Ostrowsky type inequalities; exponentially convex functions; log-convex functions; means

\section{Introduction}

For fixed $m \geq 2$, let $\mathbf{x}=\left(x_{1}, \ldots, x_{m}\right)$ and $\mathbf{y}=\left(y_{1}, \ldots, y_{m}\right)$ denote two $m$-tuples. Let

$$
\begin{array}{ll}
x_{[1]} \geq x_{[2]} \geq \cdots \geq x_{[m]}, & y_{[1]} \geq y_{[2]} \geq \cdots \geq y_{[m]}, \\
x_{(1)} \leq x_{(2)} \leq \cdots \leq x_{(m)}, & y_{(1)} \leq y_{(2)} \leq \cdots \leq y_{(m)}
\end{array}
$$

be their ordered components. We say that $\mathbf{x}$ majorizes $\mathbf{y}$ or $\mathbf{y}$ is majorized by $\mathbf{x}$ and write

$$
\mathbf{y} \prec \mathbf{x}
$$

if

$$
\begin{aligned}
& \sum_{i=1}^{k} y_{[i]} \leq \sum_{i=1}^{k} x_{[i]}, \quad k=1, \ldots, m-1, \\
& \sum_{i=1}^{m} y_{i}=\sum_{i=1}^{m} x_{i} .
\end{aligned}
$$

Note that (1.1) is equivalent to

$$
\sum_{i=m-k+1}^{m} y_{(i)} \leq \sum_{i=m-k+1}^{m} x_{(i)}, \quad k=1, \ldots, m-1 .
$$

(0) 2016 Agarwal et al. This article is distributed under the terms of the Creative Commons Attribution 4.0 International License (http://creativecommons.org/licenses/by/4.0/), which permits unrestricted use, distribution, and reproduction in any medium, provided you give appropriate credit to the original author(s) and the source, provide a link to the Creative Commons license, and indicate if changes were made. 
The following notion of Schur-convexity generalizes the definition of a convex function via the notion of majorization.

A function $F: S \subseteq \mathbb{R}^{m} \rightarrow \mathbb{R}$ is called Schur-convex on $S$ if

$$
F(\mathbf{y}) \leq F(\mathbf{x})
$$

for every $\mathbf{x}, \mathbf{y} \in S$ such that

$$
\mathbf{y} \prec \mathbf{x} .
$$

A relation between a one-dimensional convex function and an $m$-dimensional Schurconvex function is included in the following majorization theorem proved by Hardy et al. (see [1], [2], p.333).

Theorem 1 (Majorization theorem) Let $I \subset \mathbb{R}$ be an interval and $\mathbf{x}=\left(x_{1}, \ldots, x_{m}\right), \mathbf{y}=$ $\left(y_{1}, \ldots, y_{m}\right) \in I^{m}$. Let $f: I \rightarrow \mathbb{R}$ be continuous function. Then a function $F: I^{m} \rightarrow \mathbb{R}$, defined by

$$
F(\mathbf{x})=\sum_{i=1}^{m} f\left(x_{i}\right)
$$

is Schur-convex on $I^{m}$ iff is convex on I.

The following theorem gives a weighted generalization of the majorization theorem (see [3], [2], p.323).

Theorem 2 (Fuchs' theorem) Let $\mathbf{x}=\left(x_{1}, \ldots, x_{m}\right), \mathbf{y}=\left(y_{1}, \ldots, y_{m}\right) \in I^{m}$ be two decreasing $m$-tuples and $\mathbf{p}=\left(p_{1}, \ldots, p_{m}\right)$ be a real $m$-tuple such that

$$
\begin{aligned}
\sum_{i=1}^{k} p_{i} y_{i} & \leq \sum_{i=1}^{k} p_{i} x_{i}, \quad k=1, \ldots, m-1, \\
\sum_{i=1}^{m} p_{i} y_{i} & =\sum_{i=1}^{m} p_{i} x_{i} .
\end{aligned}
$$

Then for every continuous convex function $f: I \rightarrow \mathbb{R}$, we have

$$
\sum_{i=1}^{m} p_{i} f\left(y_{i}\right) \leq \sum_{i=1}^{m} p_{i} f\left(x_{i}\right)
$$

The Jensen inequality in the form

$$
f\left(\frac{1}{P_{m}} \sum_{i=1}^{m} p_{i} x_{i}\right) \leq \frac{1}{P_{m}} \sum_{i=1}^{m} p_{i} f\left(x_{i}\right)
$$

for a convex function $f$, where $\mathbf{p}=\left(p_{1}, \ldots, p_{m}\right)$ is a nonnegative $m$-tuple such that $P_{m}=$ $\sum_{i=1}^{m} p_{i}>0$, can be obtained as a special case of the previous result putting $y_{1}=y_{2}=\cdots=$ $y_{m}=\frac{1}{P_{m}} \sum_{i=1}^{m} p_{i} x_{i}$. 
A natural problem of interest is the extension of the notation from $m$-tuples (vectors) to $m \times l$ matrices $\mathbf{A}=\left(a_{i j}\right) \in \mathcal{M}_{m l}(\mathbb{R})$. Thus we introduce the notion of row stochastic and double stochastic matrices.

A matrix $\mathbf{A}=\left(a_{i j}\right) \in \mathcal{M}_{m l}(\mathbb{R})$ is called row stochastic if all of its entries are greater or equal to zero, i.e. $a_{i j} \geq 0$ for $i=1, \ldots, m, j=1, \ldots, l$ and the sum of the entries in each row is equal to 1 , i.e. $\sum_{j=1}^{l} a_{i j}=1$ for $i=1, \ldots, m$. A square matrix $\mathbf{A}=\left(a_{i j}\right) \in \mathcal{M}_{l}(\mathbb{R})$ is called double stochastic if all of its entries are greater or equal to zero (nonnegative), i.e. $a_{i j} \geq 0$ for $i, j=1, \ldots, l$, and the sum of the entries in each column and each row is equal to 1 , i.e. $\sum_{i=1}^{l} a_{i j}=1$ for $j=1, \ldots, l$ and $\sum_{j=1}^{l} a_{i j}=1$ for $i=1, \ldots, l$.

It is well known that, for $\mathbf{x}, \mathbf{y} \in \mathbb{R}^{l}$,

$$
\mathbf{y} \prec \mathbf{x} \text { if and only if } \mathbf{y}=\mathbf{x A}
$$

for some double stochastic matrix $\mathbf{A} \in \mathcal{M}_{l}(\mathbb{R})$.

The next generalization was obtained by Sherman (see $[4,5])$.

Theorem 3 (Sherman's theorem) Let $\mathbf{x}=\left(x_{1}, \ldots, x_{l}\right) \in[\alpha, \beta]^{l}, \mathbf{y}=\left(y_{1}, \ldots, y_{m}\right) \in[\alpha, \beta]^{m}, \mathbf{a}=$ $\left(a_{1}, \ldots, a_{l}\right) \in[0, \infty)^{l}, \mathbf{b}=\left(b_{1}, \ldots, b_{m}\right) \in[0, \infty)^{m}$ and

$$
\mathbf{y}=\mathbf{x} \mathbf{A}^{T} \text { and } \mathbf{a}=\mathbf{b} \mathbf{A}
$$

for some row stochastic matrix $\mathbf{A}=\left(a_{i j}\right) \in \mathcal{M}_{m l}(\mathbb{R})$. Then for every convex function $f$ : $[\alpha, \beta] \rightarrow \mathbb{R}$ we have

$$
\sum_{i=1}^{m} b_{i} f\left(y_{i}\right) \leq \sum_{j=1}^{l} a_{j} f\left(x_{j}\right) .
$$

Remark 1 In a special case, from Sherman's theorem we get Fuchs' theorem. When $m=l$, and all weights $b_{i}$ and $a_{j}$ are equal and nonnegative, the condition $\mathbf{a}=\mathbf{b A}$ ensures the stochasticity on columns, so in that case we deal with doubly stochastic matrices.

In 1929, Lidstone [6] introduced a generalization of Taylor's series: it approximates a given function in the neighborhood of two points instead of one. This series includes the polynomials later called Lidstone's polynomials. These polynomials have been studied in the work of Boas [7], Poritsky [8], Widder [9], and others. See also [10].

Definition 1 Let $f \in C^{\infty}([0,1])$, then the Lidstone series has the form

$$
\sum_{k=0}^{\infty}\left(f^{(2 k)}(0) \Lambda_{k}(1-x)+f^{(2 k)}(1) \Lambda_{k}(x)\right),
$$

where $\Lambda_{k}$ is polynomial of degree $2 n+1$ defined by the relations

$$
\begin{aligned}
& \Lambda_{0}(t)=t, \\
& \Lambda_{n}^{\prime \prime}(t)=\Lambda_{n-1}(t), \\
& \Lambda_{n}(0)=\Lambda_{n}(1)=0, \quad n \geq 1 .
\end{aligned}
$$


In [9], Widder proved the following fundamental lemma.

Lemma 1 If $f \in C^{2 n}([0,1])$, then

$$
f(t)=\sum_{k=0}^{n-1}\left(f^{(2 k)}(0) \Lambda_{k}(1-t)+f^{(2 k)}(1) \Lambda_{k}(t)\right)+\int_{0}^{1} G_{n}(t, s) f^{(2 n)}(s) d s,
$$

where

$$
G_{1}(t, s)=G(t, s)= \begin{cases}(t-1) s, & s \leq t \\ (s-1) t, & t \leq s\end{cases}
$$

is a homogeneous Green's function of the differential operator $\frac{d^{2}}{d s^{2}}$ on $[0,1]$ and with the successive iterates of $G(t, s)$,

$$
G_{n}(t, s)=\int_{0}^{1} G_{1}(t, p) G_{n-1}(p, s) d p, \quad n \geq 2 .
$$

Remark 2 Green's function $G$ defined by (1.7) is convex and continuous with respect to both variables $s$ and $t$.

The Lidstone polynomial can be expressed in terms of $G_{n}(t, s)$ as

$$
\Lambda_{n}(t)=\int_{0}^{1} G_{n}(t, s) s d s
$$

To complete the Introduction, we state a definition of the divided differences and $n$ convexity (see for example [2]).

Definition 2 The divided difference of order $n, n \in \mathbb{N}$, of the function $f:[\alpha, \beta] \rightarrow \mathbb{R}$ at mutually different points $x_{0}, x_{1}, \ldots, x_{n} \in[\alpha, \beta]$ is defined recursively by

$$
\begin{aligned}
& {\left[x_{i} ; f\right]=f\left(x_{i}\right), \quad i=0, \ldots, n,} \\
& {\left[x_{0}, \ldots, x_{n} ; f\right]=\frac{\left[x_{1}, \ldots, x_{n} ; f\right]-\left[x_{0}, \ldots, x_{n-1} ; f\right]}{x_{n}-x_{0}} .}
\end{aligned}
$$

The value of $\left[x_{0}, \ldots, x_{n} ; f\right]$ is independent of the order of the points $x_{0}, \ldots, x_{n}$.

This definition may be extended to include the case in which some or all the points coincide. Assuming that $f^{(j-1)}(x)$ exists, we define

$$
[\underbrace{x, \ldots, x ; f]}_{j \text {-times }}=\frac{f^{(j-1)}(x)}{(j-1) !} .
$$

Definition 3 A function $f:[\alpha, \beta] \rightarrow \mathbb{R}$ is $n$-convex, $n \geq 0$, if for all choices of $(n+1)$ distinct points $x_{i} \in[\alpha, \beta], i=0, \ldots, n$, the inequality

$$
\left[x_{0}, x_{1}, \ldots, x_{n} ; f\right] \geq 0
$$

holds. 
From Definition 3, it follows that 2-convex functions are just convex functions. Furthermore, 1-convex functions are increasing functions and 0-convex functions are nonnegative functions.

\section{Main results}

First we prove an identity related to a generalization of Sherman's inequality using Lidstone's interpolating polynomial.

Theorem 4 Let $\mathbf{x}=\left(x_{1}, \ldots, x_{l}\right) \in[\alpha, \beta]^{l}, \mathbf{y}=\left(y_{1}, \ldots, y_{m}\right) \in[\alpha, \beta]^{m}, \mathbf{a}=\left(a_{1}, \ldots, a_{l}\right) \in \mathbb{R}^{l}, \mathbf{b}=$ $\left(b_{1}, \ldots, b_{m}\right) \in \mathbb{R}^{m}$ and $\phi \in C^{2 n}([\alpha, \beta])$. Then

$$
\begin{aligned}
& \sum_{j=1}^{l} a_{j} \phi\left(x_{j}\right)-\sum_{i=1}^{m} b_{i} \phi\left(y_{i}\right) \\
& =\sum_{k=0}^{n-1}(\beta-\alpha)^{2 k} \phi^{(2 k)}(\alpha)\left[\sum_{j=1}^{l} a_{j} \Lambda_{k}\left(\frac{\beta-x_{j}}{\beta-\alpha}\right)-\sum_{i=1}^{m} b_{i} \Lambda_{k}\left(\frac{\beta-y_{i}}{\beta-\alpha}\right)\right] \\
& \quad+\sum_{k=0}^{n-1}(\beta-\alpha)^{2 k} \phi^{(2 k)}(\beta)\left[\sum_{j=1}^{l} a_{j} \Lambda_{k}\left(\frac{x_{j}-\alpha}{\beta-\alpha}\right)-\sum_{i=1}^{m} b_{i} \Lambda_{k}\left(\frac{y_{i}-\alpha}{\beta-\alpha}\right)\right] \\
& \quad+(\beta-\alpha)^{2 n-1} \int_{\alpha}^{\beta}\left[\sum_{j=1}^{l} a_{j} G_{n}\left(\frac{x_{j}-\alpha}{\beta-\alpha}, \frac{t-\alpha}{\beta-\alpha}\right)-\sum_{i=1}^{m} b_{i} G_{n}\left(\frac{y_{i}-\alpha}{\beta-\alpha}, \frac{t-\alpha}{\beta-\alpha}\right)\right] \\
& \quad \times \phi^{(2 n)}(t) d t .
\end{aligned}
$$

Proof By Widder's lemma, we can represent every function $\phi \in C^{2 n}([\alpha, \beta])$ in the form

$$
\begin{aligned}
\phi(x)= & \sum_{k=0}^{n-1}(\beta-\alpha)^{2 k}\left[\phi^{(2 k)}(\alpha) \Lambda_{k}\left(\frac{\beta-x}{\beta-\alpha}\right)+\phi^{(2 k)}(\beta) \Lambda_{k}\left(\frac{x-\alpha}{\beta-\alpha}\right)\right] \\
& +(\beta-\alpha)^{2 n-1} \int_{\alpha}^{\beta} G_{n}\left(\frac{x-\alpha}{\beta-\alpha}, \frac{s-\alpha}{\beta-\alpha}\right) \phi^{(2 n)}(s) d s .
\end{aligned}
$$

Using (2.2), we have

$$
\begin{aligned}
& \sum_{j=1}^{l} a_{j} \phi\left(x_{j}\right)-\sum_{i=1}^{m} b_{i} \phi\left(y_{i}\right) \\
& =\sum_{j=1}^{l} a_{j}\left\{\sum_{k=0}^{n-1}(\beta-\alpha)^{2 k}\left[\phi^{(2 k)}(\alpha) \Lambda_{k}\left(\frac{\beta-x_{j}}{\beta-\alpha}\right)+\phi^{(2 k)}(\beta) \Lambda_{k}\left(\frac{x_{j}-\alpha}{\beta-\alpha}\right)\right]\right\} \\
& +\sum_{j=1}^{l} a_{j}\left[(\beta-\alpha)^{2 n-1} \int_{\alpha}^{\beta} G_{n}\left(\frac{x_{j}-\alpha}{\beta-\alpha}, \frac{t-\alpha}{\beta-\alpha}\right) \phi^{(2 n)}(t) d t\right] \\
& \quad-\sum_{i=1}^{m} b_{i}\left\{\sum_{k=0}^{n-1}(\beta-\alpha)^{2 k}\left[\phi^{(2 k)}(\alpha) \Lambda_{k}\left(\frac{\beta-y_{i}}{\beta-\alpha}\right)+\phi^{(2 k)}(\beta) \Lambda_{k}\left(\frac{y_{i}-\alpha}{\beta-\alpha}\right)\right]\right\} \\
& \quad-\sum_{i=1}^{m} b_{i}\left[(\beta-\alpha)^{2 n-1} \int_{\alpha}^{\beta} G_{n}\left(\frac{y_{i}-\alpha}{\beta-\alpha}, \frac{t-\alpha}{\beta-\alpha}\right) \phi^{(2 n)}(t) d t\right] .
\end{aligned}
$$


By an easy calculation, from (2.3) we get (2.1).

Using the previous result, we get the following generalizations of Sherman's theorem for $2 n$-convex functions.

Theorem 5 Let $n \in \mathbb{N}, \mathbf{x}=\left(x_{1}, \ldots, x_{l}\right) \in[\alpha, \beta]^{l}, \mathbf{y}=\left(y_{1}, \ldots, y_{m}\right) \in[\alpha, \beta]^{m}, \mathbf{a}=\left(a_{1}, \ldots, a_{l}\right) \in$ $\mathbb{R}^{l}$ and $\mathbf{b}=\left(b_{1}, \ldots, b_{m}\right) \in \mathbb{R}^{m}$. If for all $t \in[\alpha, \beta]$ we have

$$
\sum_{i=1}^{m} b_{i} G_{n}\left(\frac{y_{i}-\alpha}{\beta-\alpha}, \frac{t-\alpha}{\beta-\alpha}\right) \leq \sum_{j=1}^{l} a_{j} G_{n}\left(\frac{x_{j}-\alpha}{\beta-\alpha}, \frac{t-\alpha}{\beta-\alpha}\right),
$$

then for every $2 n$-convex function $\phi:[\alpha, \beta] \rightarrow \mathbb{R}$, we have

$$
\begin{aligned}
& \sum_{j=1}^{l} a_{j} \phi\left(x_{j}\right)-\sum_{i=1}^{m} b_{i} \phi\left(y_{i}\right) \\
& \geq \sum_{k=0}^{n-1}(\beta-\alpha)^{2 k} \phi^{(2 k)}(\alpha)\left[\sum_{j=1}^{l} a_{j} \Lambda_{k}\left(\frac{\beta-x_{j}}{\beta-\alpha}\right)-\sum_{i=1}^{m} b_{i} \Lambda_{k}\left(\frac{\beta-y_{i}}{\beta-\alpha}\right)\right] \\
& \quad+\sum_{k=0}^{n-1}(\beta-\alpha)^{2 k} \phi^{(2 k)}(\beta)\left[\sum_{j=1}^{l} a_{j} \Lambda_{k}\left(\frac{x_{j}-\alpha}{\beta-\alpha}\right)-\sum_{i=1}^{m} b_{i} \Lambda_{k}\left(\frac{y_{i}-\alpha}{\beta-\alpha}\right)\right] .
\end{aligned}
$$

If the reverse inequality in (2.4) holds, then the reverse inequality in (2.5) holds.

Proof Since a function $\phi$ is $2 n$-convex, we may assume without loss of generality that $\phi$ is $2 n$-times differentiable and $\phi^{(2 n)}(t) \geq 0, t \in[\alpha, \beta]$ (see [2], p.16).

Using this fact and the assumption (2.4), applying Theorem 4 we obtain (2.5).

Under the assumptions of Sherman's theorem the following generalizations are valid.

Theorem 6 Let $n \in \mathbb{N}, \mathbf{x}=\left(x_{1}, \ldots, x_{l}\right) \in[\alpha, \beta]^{l}, \mathbf{y}=\left(y_{1}, \ldots, y_{m}\right) \in[\alpha, \beta]^{m}, \mathbf{a}=\left(a_{1}, \ldots, a_{l}\right) \in$ $[0, \infty)^{l}, \mathbf{b}=\left(b_{1}, \ldots, b_{m}\right) \in[0, \infty)^{m}$ and let (1.4) holds for some row stochastic matrix $\mathbf{A}=$ $\left(a_{i j}\right) \in \mathcal{M}_{m l}(\mathbb{R})$.

(i) If $n$ is odd, then for every $2 n$-convex function $\phi:[\alpha, \beta] \rightarrow \mathbb{R}$, we have

$$
\begin{aligned}
& \sum_{j=1}^{l} a_{j} \phi\left(x_{j}\right)-\sum_{i=1}^{m} b_{i} \phi\left(y_{i}\right) \\
& \geq \sum_{k=1}^{n-1}(\beta-\alpha)^{2 k} \phi^{(2 k)}(\alpha)\left[\sum_{j=1}^{l} a_{j} \Lambda_{k}\left(\frac{\beta-x_{j}}{\beta-\alpha}\right)-\sum_{i=1}^{m} b_{i} \Lambda_{k}\left(\frac{\beta-y_{i}}{\beta-\alpha}\right)\right] \\
& \quad+\sum_{k=1}^{n-1}(\beta-\alpha)^{2 k} \phi^{(2 k)}(\beta)\left[\sum_{j=1}^{l} a_{j} \Lambda_{k}\left(\frac{x_{j}-\alpha}{\beta-\alpha}\right)-\sum_{i=1}^{m} b_{i} \Lambda_{k}\left(\frac{y_{i}-\alpha}{\beta-\alpha}\right)\right] .
\end{aligned}
$$

Moreover, if $\phi^{(2 k)}(\alpha) \geq 0, \phi^{(2 k)}(\beta) \geq 0$ for $k=1,3, \ldots, n-2$ and $\phi^{(2 k)}(\alpha) \leq 0$, $\phi^{(2 k)}(\beta) \leq 0$ for $k=2,4, \ldots, n-1$, then

$$
\sum_{i=1}^{m} b_{i} \phi\left(y_{i}\right) \leq \sum_{j=1}^{l} a_{j} \phi\left(x_{j}\right)
$$


(ii) If $n$ is even, then for every $2 n$-convex function $\phi:[\alpha, \beta] \rightarrow \mathbb{R}$, the reverse inequality in (2.6) holds. Moreover, if $\phi^{(2 k)}(\alpha) \leq 0, \phi^{(2 k)}(\beta) \leq 0$ for $k=1,3, \ldots, n-1$ and $\phi^{(2 k)}(\alpha) \geq 0, \phi^{(2 k)}(\beta) \geq 0$ for $k=2,4, \ldots, n-2$, then the reverse inequality in (2.7) holds.

Proof (i) From (1.7), it follows that $G_{1}(t, s) \leq 0$ for $0 \leq t, s \leq 1$.

From (1.8), it follows that $G_{n}(t, s) \leq 0$ for odd $n$ and $G_{n}(t, s) \geq 0$ for even $n$.

Now, since $G_{1}$ is convex and $G_{n-1}$ is nonnegative for odd $n$, using (1.8), we conclude that $G_{n}$ is convex in first variable if $n$ is odd. Similarly, we see that $G_{n}$ is concave in the first variable if $n$ is even.

Hence, if $n$ is odd, then by Sherman's theorem we have

$$
\sum_{i=1}^{m} b_{i} G_{n}\left(\frac{y_{i}-\alpha}{\beta-\alpha}, \frac{t-\alpha}{\beta-\alpha}\right) \leq \sum_{j=1}^{l} a_{j} G_{n}\left(\frac{x_{j}-\alpha}{\beta-\alpha}, \frac{t-\alpha}{\beta-\alpha}\right) .
$$

Therefore, in this case, by Theorem 5 , the inequality (2.6) holds.

Using the representation (1.9), we also conclude that $\Lambda_{n}$ is convex if $n$ is odd and concave if $n$ is even. Then, by Sherman's theorem, we have

$$
\sum_{i=1}^{m} b_{i} \Lambda_{k}\left(\frac{y_{i}-\alpha}{\beta-\alpha}\right) \leq \sum_{j=1}^{l} a_{j} \Lambda_{k}\left(\frac{x_{j}-\alpha}{\beta-\alpha}\right)
$$

if $k$ is odd and the reverse inequality in (2.8) holds if $k$ is even.

Moreover, if $\phi^{(2 k)}(\alpha) \geq 0, \phi^{(2 k)}(\beta) \geq 0$ for $k=1,3, \ldots, n-2$ and $\phi^{(2 k)}(\alpha) \leq 0, \phi^{(2 k)}(\beta) \leq 0$ for $k=2,4, \ldots, n-1$, then the right-hand side in (2.5) is nonnegative and (2.7) immediately follows.

(ii) Similar to part (i).

Remark 3 Note that as a special case of Theorem 5 we get Sherman's theorem. For $n=1$, with the assumptions (1.4) and using the fact that $G_{1}(\cdot, t)=G(\cdot, t), t \in[0,1]$, is continuous and convex on $[0,1]$, we have

$$
\begin{aligned}
\sum_{i=1}^{m} b_{i} G\left(\frac{y_{i}-\alpha}{\beta-\alpha}, \frac{t-\alpha}{\beta-\alpha}\right) & =\sum_{i=1}^{m} b_{i} G\left(\frac{\sum_{j=1}^{l} x_{j} a_{i j}-\alpha}{\beta-\alpha}, \frac{t-\alpha}{\beta-\alpha}\right) \\
& \leq \sum_{i=1}^{m} b_{i} \sum_{j=1}^{l} a_{i j} G\left(\frac{x_{j}-\alpha}{\beta-\alpha}, \frac{t-\alpha}{\beta-\alpha}\right) \\
& =\sum_{j=1}^{l}\left(\sum_{i=1}^{m} b_{i} a_{i j}\right) G\left(\frac{x_{j}-\alpha}{\beta-\alpha}, \frac{t-\alpha}{\beta-\alpha}\right) \\
& =\sum_{j=1}^{l} a_{j} G\left(\frac{x_{j}-\alpha}{\beta-\alpha}, \frac{t-\alpha}{\beta-\alpha}\right)
\end{aligned}
$$

i.e. the assumption (2.4) holds. Then by Theorem 5 , the inequality (1.5) immediately follows. 


\section{Bounds for identities related to generalizations of Sherman's inequality}

For two Lebesgue integrable functions $f, g:[\alpha, \beta] \rightarrow \mathbb{R}$, we consider the Čebyšev functional:

$$
T(f, g):=\frac{1}{\beta-\alpha} \int_{\alpha}^{\beta} f(t) g(t) d t-\frac{1}{\beta-\alpha} \int_{\alpha}^{\beta} f(t) d t \cdot \frac{1}{\beta-\alpha} \int_{\alpha}^{\beta} g(t) d t .
$$

We use the following two theorems, proved in [11], to obtain generalizations of the results from the previous section.

Theorem 7 Let $f:[\alpha, \beta] \rightarrow \mathbb{R}$ be Lebesgue integrable and $g:[\alpha, \beta] \rightarrow \mathbb{R}$ be absolutely continuous with $(\cdot-\alpha)(\beta-\cdot)\left(g^{\prime}\right)^{2} \in L[\alpha, \beta]$. Then

$$
|T(f, g)| \leq \frac{1}{\sqrt{2}}[T(f, f)]^{\frac{1}{2}} \frac{1}{\sqrt{\beta-\alpha}}\left(\int_{\alpha}^{\beta}(x-\alpha)(\beta-x)\left[g^{\prime}(x)\right]^{2} d x\right)^{\frac{1}{2}} .
$$

The constant $\frac{1}{\sqrt{2}}$ in (3.1) is the best possible.

Theorem 8 Let $g:[\alpha, \beta] \rightarrow \mathbb{R}$ be monotonic nondecreasing and $f:[\alpha, \beta] \rightarrow \mathbb{R}$ be absolutely continuous with $f^{\prime} \in L_{\infty}[\alpha, \beta]$. Then

$$
|T(f, g)| \leq \frac{1}{2(\beta-\alpha)}\left\|f^{\prime}\right\|_{\infty} \int_{\alpha}^{\beta}(x-\alpha)(\beta-x) d g(x) .
$$

The constant $\frac{1}{2}$ in (3.2) is the best possible.

For the sake of simplicity and to avoid an overload of notations, we define two functions as follows.

Let $\mathbf{x}=\left(x_{1}, \ldots, x_{l}\right) \in[\alpha, \beta]^{l}, \mathbf{y}=\left(y_{1}, \ldots, y_{m}\right) \in[\alpha, \beta]^{m}, \mathbf{a}=\left(a_{1}, \ldots, a_{l}\right) \in \mathbb{R}^{l}, \mathbf{b}=\left(b_{1}, \ldots\right.$, $\left.b_{m}\right) \in \mathbb{R}^{m}$ and the function $G_{n}$ be defined as in (1.7) and (1.8). The function $\mathcal{R}:[\alpha, \beta] \rightarrow \mathbb{R}$ is defined by

$$
\mathcal{R}(t)=\sum_{j=1}^{l} a_{j} G_{n}\left(\frac{x_{j}-\alpha}{\beta-\alpha}, \frac{t-\alpha}{\beta-\alpha}\right)-\sum_{i=1}^{m} b_{i} G_{n}\left(\frac{y_{i}-\alpha}{\beta-\alpha}, \frac{t-\alpha}{\beta-\alpha}\right) .
$$

Using the Čebyšev functional we obtain a bound for the identity (2.1) related to a generalization of Sherman's inequality.

Theorem 9 Let $\phi:[\alpha, \beta] \rightarrow \mathbb{R}$ be such that $\phi \in C^{2 n}([\alpha, \beta])$ for $n \in \mathbb{N}$ with $(\cdot-\alpha)(\beta-$ $\cdot)\left(\phi^{(2 n+1)}\right)^{2} \in L[\alpha, \beta]$. Let the function $\mathcal{R}$ be defined as in (3.3). Then

$$
\begin{aligned}
& \sum_{j=1}^{l} a_{j} \phi\left(x_{j}\right)-\sum_{i=1}^{m} b_{i} \phi\left(y_{i}\right) \\
& =\sum_{k=0}^{n-1}(\beta-\alpha)^{2 k} \phi^{(2 k)}(\alpha)\left[\sum_{j=1}^{l} a_{j} \Lambda_{k}\left(\frac{\beta-x_{j}}{\beta-\alpha}\right)-\sum_{i=1}^{m} b_{i} \Lambda_{k}\left(\frac{\beta-y_{i}}{\beta-\alpha}\right)\right] \\
& \quad+\sum_{k=0}^{n-1}(\beta-\alpha)^{2 k} \phi^{(2 k)}(\beta)\left[\sum_{j=1}^{l} a_{j} \Lambda_{k}\left(\frac{x_{j}-\alpha}{\beta-\alpha}\right)-\sum_{i=1}^{m} b_{i} \Lambda_{k}\left(\frac{y_{i}-\alpha}{\beta-\alpha}\right)\right] \\
& \quad+(\beta-\alpha)^{2 n-2}\left[\phi^{(2 n-1)}(\beta)-\phi^{(2 n-1)}(\alpha)\right] \int_{\alpha}^{\beta} \mathcal{R}(t) d t+\kappa_{n}^{1}(\phi ; \alpha, \beta),
\end{aligned}
$$


where the remainder $\kappa_{n}^{1}(\phi ; \alpha, \beta)$ satisfies the estimation

$$
\left|\kappa_{n}^{1}(\phi ; \alpha, \beta)\right| \leq \frac{(\beta-\alpha)^{2 n-\frac{1}{2}}}{\sqrt{2}}[T(\mathcal{R}, \mathcal{R})]^{\frac{1}{2}}\left(\int_{\alpha}^{\beta}(t-\alpha)(\beta-t)\left[\phi^{(2 n+1)}(t)\right]^{2} d t\right)^{\frac{1}{2}} .
$$

Proof If we apply Theorem 7 for $f \rightarrow \mathcal{R}$ and $g \rightarrow \phi^{(2 n)}$ we obtain

$$
\begin{gathered}
\left|\frac{1}{\beta-\alpha} \int_{\alpha}^{\beta} \mathcal{R}(t) \phi^{(2 n)}(t) d t-\frac{1}{\beta-\alpha} \int_{\alpha}^{\beta} \mathcal{R}(t) d t \cdot \frac{1}{\beta-\alpha} \int_{\alpha}^{\beta} \phi^{(2 n)}(t) d t\right| \\
\leq \frac{1}{\sqrt{2}}[T(\mathcal{R}, \mathcal{R})]^{\frac{1}{2}} \frac{1}{\sqrt{\beta-\alpha}}\left(\int_{\alpha}^{\beta}(t-\alpha)(\beta-t)\left[\phi^{(2 n+1)}(t)\right]^{2} d t\right)^{\frac{1}{2}} .
\end{gathered}
$$

Therefore, we have

$$
\begin{aligned}
(\beta & -\alpha)^{2 n-1} \int_{\alpha}^{\beta} \mathcal{R}(t) \phi^{(2 n)}(t) d t \\
& =(\beta-\alpha)^{2 n-2}\left[\phi^{(2 n-1)}(\beta)-\phi^{(2 n-1)}(\alpha)\right] \int_{\alpha}^{\beta} \mathcal{R}(t) d t+\kappa_{n}^{1}(\phi ; \alpha, \beta),
\end{aligned}
$$

where the remainder $\kappa_{n}^{1}(\phi ; \alpha, \beta)$ satisfies the estimation (3.5).

Now from the identity (2.1) we obtain (3.4).

Using Theorem 8 we obtain the Grüss type inequality.

Theorem 10 Let $\phi:[\alpha, \beta] \rightarrow \mathbb{R}$ be such that $\phi \in C^{2 n}([\alpha, \beta])$ for $n \in \mathbb{N}$ and $\phi^{(2 n+1)} \geq 0$ on $[\alpha, \beta]$. Let the function $\mathcal{R}$ be defined as in (3.3). Then the representation (3.4) and the remainder $\kappa_{n}^{1}(\phi ; \alpha, \beta)$ satisfies the bound

$$
\begin{aligned}
& \left|\kappa_{n}^{1}(\phi ; \alpha, \beta)\right| \\
& \quad \leq(\beta-\alpha)^{2 n-1}\left\|\mathcal{R}^{\prime}\right\|_{\infty}\left[\frac{\phi^{(2 n-1)}(\beta)+\phi^{(2 n-1)}(\alpha)}{2}-\frac{\phi^{(2 n-2)}(\beta)-\phi^{(2 n-2)}(\alpha)}{\beta-\alpha}\right] .
\end{aligned}
$$

Proof Applying Theorem 8 for $f \rightarrow \mathcal{R}$ and $g \rightarrow \phi^{(2 n)}$ we obtain

$$
\begin{aligned}
& \left|\frac{1}{\beta-\alpha} \int_{\alpha}^{\beta} \mathcal{R}(t) \phi^{(2 n)}(t) d t-\frac{1}{\beta-\alpha} \int_{\alpha}^{\beta} \mathcal{R}(t) d t \cdot \frac{1}{\beta-\alpha} \int_{\alpha}^{\beta} \phi^{(2 n)}(t) d t\right| \\
& \quad \leq \frac{1}{2(\beta-\alpha)}\left\|\mathcal{R}^{\prime}\right\|_{\infty} \int_{\alpha}^{\beta}(t-\alpha)(\beta-t) \phi^{(2 n+1)}(t) d t .
\end{aligned}
$$

Since

$$
\begin{aligned}
& \int_{\alpha}^{\beta}(t-\alpha)(\beta-t) \phi^{(2 n+1)}(t) d t \\
& \quad=\int_{\alpha}^{\beta}[2 t-(\alpha+\beta)] \phi^{(2 n)}(t) d t \\
& \quad=(\beta-\alpha)\left[\phi^{(2 n-1)}(\beta)+\phi^{(2 n-1)}(\alpha)\right]-2\left[\phi^{(2 n-2)}(\beta)-\phi^{(2 n-2)}(\alpha)\right],
\end{aligned}
$$

using the identity (2.1) and the inequality (3.7) we deduce (3.6). 
We present the Ostrowsky type inequality related to generalizations of Sherman's inequality.

Theorem 11 Suppose that all assumptions of Theorem 4 hold. Assume that $(p, q)$ is a pair of conjugate exponents, that is, $1 \leq p, q \leq \infty, 1 / p+1 / q=1$. Let $\phi^{(2 n)} \in L_{p}[\alpha, \beta]$. Then

$$
\begin{aligned}
& \mid \sum_{j=1}^{l} a_{j} \phi\left(x_{j}\right)-\sum_{i=1}^{m} b_{i} \phi\left(y_{i}\right) \\
& \quad-\sum_{k=0}^{n-1}(\beta-\alpha)^{2 k} \phi^{(2 k)}(\alpha)\left[\sum_{j=1}^{l} a_{j} \Lambda_{k}\left(\frac{\beta-x_{j}}{\beta-\alpha}\right)-\sum_{i=1}^{m} b_{i} \Lambda_{k}\left(\frac{\beta-y_{i}}{\beta-\alpha}\right)\right] \\
& \quad-\sum_{k=0}^{n-1}(\beta-\alpha)^{2 k} \phi^{(2 k)}(\beta)\left[\sum_{j=1}^{l} a_{j} \Lambda_{k}\left(\frac{x_{j}-\alpha}{\beta-\alpha}\right)-\sum_{i=1}^{m} b_{i} \Lambda_{k}\left(\frac{y_{i}-\alpha}{\beta-\alpha}\right)\right] \mid \\
& \leq(\beta-\alpha)^{2 n-1}\left\|\phi^{(2 n)}\right\|_{p} \\
& \quad \times\left[\int_{\alpha}^{\beta}\left|\sum_{j=1}^{l} a_{j} G_{n}\left(\frac{x_{j}-\alpha}{\beta-\alpha}, \frac{t-\alpha}{\beta-\alpha}\right)-\sum_{i=1}^{m} b_{i} G_{n}\left(\frac{y_{i}-\alpha}{\beta-\alpha}, \frac{t-\alpha}{\beta-\alpha}\right)\right|^{q} d t\right]^{\frac{1}{q}} .
\end{aligned}
$$

The constant on the right-hand side of (3.8) is sharp for $1<p \leq \infty$ and the best possible for $p=1$.

Proof Let us denote

$$
\mathcal{S}(t)=(\beta-\alpha)^{2 n-1}\left[\sum_{j=1}^{l} a_{j} G_{n}\left(\frac{x_{j}-\alpha}{\beta-\alpha}, \frac{t-\alpha}{\beta-\alpha}\right)-\sum_{i=1}^{m} b_{i} G_{n}\left(\frac{y_{i}-\alpha}{\beta-\alpha}, \frac{t-\alpha}{\beta-\alpha}\right)\right] .
$$

Using the identity (2.1) and applying the well-known Hölder inequality, we obtain

$$
\begin{aligned}
& \mid \sum_{j=1}^{l} a_{j} \phi\left(x_{j}\right)-\sum_{i=1}^{m} b_{i} \phi\left(y_{i}\right) \\
& \quad-\sum_{k=0}^{n-1}(\beta-\alpha)^{2 k} \phi^{(2 k)}(\alpha)\left[\sum_{j=1}^{l} a_{j} \Lambda_{k}\left(\frac{\beta-x_{j}}{\beta-\alpha}\right)-\sum_{i=1}^{m} b_{i} \Lambda_{k}\left(\frac{\beta-y_{i}}{\beta-\alpha}\right)\right] \\
& \quad-\sum_{k=0}^{n-1}(\beta-\alpha)^{2 k} \phi^{(2 k)}(\beta)\left[\sum_{j=1}^{l} a_{j} \Lambda_{k}\left(\frac{x_{j}-\alpha}{\beta-\alpha}\right)-\sum_{i=1}^{m} b_{i} \Lambda_{k}\left(\frac{y_{i}-\alpha}{\beta-\alpha}\right)\right] \mid \\
& =\left|\int_{\alpha}^{\beta} \mathcal{S}(t) \phi^{(2 n)}(t) d t\right| \leq\left\|\phi^{(2 n)}\right\|_{p}\left(\int_{\alpha}^{\beta}|\mathcal{S}(t)|^{q} d t\right)^{\frac{1}{q}} .
\end{aligned}
$$

For the proof of the sharpness of the constant $\left(\int_{\alpha}^{\beta}|\mathcal{S}(t)|^{q} d t\right)^{\frac{1}{q}}$, let us find a function $\phi$ for which the equality in (3.8) is obtained.

For $1<p<\infty$ take $\phi$ to be such that

$$
\phi^{(2 n)}(t)=\operatorname{sgn} \mathcal{S}(t)|\mathcal{S}(t)|^{\frac{1}{p-1}}
$$

For $p=\infty$ take $\phi^{(2 n)}(t)=\operatorname{sgn} \mathcal{S}(t)$. 
For $p=1$ we prove that

$$
\left|\int_{\alpha}^{\beta} \mathcal{S}(t) \phi^{(2 n)}(t) d t\right| \leq \max _{t \in[\alpha, \beta]}|\mathcal{S}(t)|\left(\int_{\alpha}^{\beta}\left|\phi^{(2 n)}(t)\right| d t\right)
$$

is the best possible inequality.

Suppose that $|\mathcal{S}(t)|$ attains its maximum at $t_{0} \in[\alpha, \beta]$.

First we assume $\mathcal{S}\left(t_{0}\right)>0$. For $\varepsilon$ small enough we define $\phi_{\varepsilon}(t)$ by

$$
\phi_{\varepsilon}(t)= \begin{cases}0, & \alpha \leq t \leq t_{0}, \\ \frac{1}{\varepsilon n !}\left(t-t_{0}\right)^{n}, & t_{0} \leq t \leq t_{0}+\varepsilon, \\ \frac{1}{n !}\left(t-t_{0}\right)^{n-1}, & t_{0}+\varepsilon \leq t \leq \beta .\end{cases}
$$

Then for $\varepsilon$ small enough

$$
\begin{aligned}
\left|\int_{\alpha}^{\beta} \mathcal{S}(t) \phi^{(2 n)}(t) d t\right| & =\left|\int_{t_{0}}^{t_{0}+\varepsilon} \mathcal{S}(t) \frac{1}{\varepsilon} d t\right| \\
& =\frac{1}{\varepsilon} \int_{t_{0}}^{t_{0}+\varepsilon} \mathcal{S}(t) d t .
\end{aligned}
$$

Now from the inequality (3.9) we have

$$
\frac{1}{\varepsilon} \int_{t_{0}}^{t_{0}+\varepsilon} \mathcal{S}(t) d t \leq \mathcal{S}\left(t_{0}\right) \int_{t_{0}}^{t_{0}+\varepsilon} \frac{1}{\varepsilon} d t=\mathcal{S}\left(t_{0}\right)
$$

Since

$$
\lim _{\varepsilon \rightarrow 0} \frac{1}{\varepsilon} \int_{t_{0}}^{t_{0}+\varepsilon} \mathcal{S}(t) d t=\mathcal{S}\left(t_{0}\right)
$$

the statement follows.

In the case $\mathcal{S}\left(t_{0}\right)<0$, we define $\phi_{\varepsilon}(t)$ by

$$
\phi_{\varepsilon}(t)= \begin{cases}\frac{1}{n !}\left(t-t_{0}-\varepsilon\right)^{n-1}, & \alpha \leq t \leq t_{0} \\ -\frac{1}{\varepsilon n !}\left(t-t_{0}-\varepsilon\right)^{n}, & t_{0} \leq t \leq t_{0}+\varepsilon \\ 0, & t_{0}+\varepsilon \leq t \leq \beta\end{cases}
$$

and the rest of the proof is the same as above.

\section{Mean value theorems and exponential convexity}

In this section, we present mean-value theorems of Lagrange and Cauchy type using results from the previous section. We also use the so-called exponential convexity method, established in [12], in order to interpret our results in the form of exponentially convex functions or in the special case logarithmically convex functions. For some related results see also $[13,14]$.

Motivated by the inequality (2.5), we define the linear functional as follows. 
Under the assumptions of Theorem 5, equipped with condition (2.4), we define

$$
\begin{aligned}
A(\phi)= & \sum_{j=1}^{l} a_{j} \phi\left(x_{j}\right)-\sum_{i=1}^{m} b_{i} \phi\left(y_{i}\right) \\
& -\sum_{k=0}^{n-1}(\beta-\alpha)^{2 k} \phi^{(2 k)}(\alpha)\left[\sum_{j=1}^{l} a_{j} \Lambda_{k}\left(\frac{\beta-x_{j}}{\beta-\alpha}\right)-\sum_{i=1}^{m} b_{i} \Lambda_{k}\left(\frac{\beta-y_{i}}{\beta-\alpha}\right)\right] \\
& -\sum_{k=0}^{n-1}(\beta-\alpha)^{2 k} \phi^{(2 k)}(\beta)\left[\sum_{j=1}^{l} a_{j} \Lambda_{k}\left(\frac{x_{j}-\alpha}{\beta-\alpha}\right)-\sum_{i=1}^{m} b_{i} \Lambda_{k}\left(\frac{y_{i}-\alpha}{\beta-\alpha}\right)\right] .
\end{aligned}
$$

Remark 4 It should be noticed that if $\phi:[\alpha, \beta] \rightarrow \mathbb{R}$ is $2 n$-convex, by Theorem 5 , we have

$$
A(\phi) \geq 0 \text {. }
$$

Theorem 12 Let $A$ be the linear functional defined as in (4.1). Let $\phi \in C^{2 n}([\alpha, \beta])$. Then there exists $\xi \in[\alpha, \beta]$ such that

$$
A(\phi)=\phi^{(2 n)}(\xi) A\left(\varphi_{0}\right)
$$

where $\varphi_{0}(x)=x^{2 n} /(2 n) !$.

Proof Our proof proceeds similarly to the proof of Theorem 1.19 in [12].

Theorem 13 Let $A$ be the linear functional defined as in (4.1). Let $\phi, \psi \in C^{2 n}([\alpha, \beta])$. Then there exists $\xi \in[\alpha, \beta]$ such that

$$
\frac{\phi^{(2 n)}(\xi)}{\psi^{(2 n)}(\xi)}=\frac{A(\phi)}{A(\psi)},
$$

assuming neither of the denominators is equal to zero.

Proof This is standard proof as in the Cauchy mean-value theorem.

$\operatorname{Remark} 5$ If $\frac{\phi^{(2 n)}}{\psi^{(2 n)}}$ is an invertible function, then from (4.2) it follows that

$$
\xi=\left(\frac{\phi^{(2 n)}}{\psi^{(2 n)}}\right)^{-1}\left(\frac{A(\phi)}{A(\psi)}\right) .
$$

Throughout the rest of this paper, $I$ denotes an open interval in $\mathbb{R}$.

The notation of $n$-exponential convexity is introduced in [14].

Definition 4 For fixed $n \in \mathbb{N}$, a function $f: I \rightarrow \mathbb{R}$ is $n$-exponentially convex in the Jensen sense on $I$ if

$$
\sum_{i, j=1}^{n} p_{i} p_{j} f\left(\frac{x_{i}+x_{j}}{2}\right) \geq 0
$$

holds for all choices $p_{i} \in \mathbb{R}$ and $x_{i} \in I, i=1, \ldots, n$. 
A function $f: I \rightarrow \mathbb{R}$ is $n$-exponentially convex on $I$ if it is $n$-exponentially convex in the Jensen sense and continuous on $I$.

Remark 6 From Definition 4 it follows that 1-exponentially convex functions in the Jensen sense are exactly nonnegative functions. Also, $n$-exponentially convex functions in the Jensen sense are $k$-exponentially convex in the Jensen sense for every $k \in \mathbb{N}, k \leq n$.

Definition 5 A function $f: I \rightarrow \mathbb{R}$ is exponentially convex in the Jensen sense on $I$ if it is $n$-exponentially convex in the Jensen sense for all $n \in \mathbb{N}$.

Definition 6 A function $f: I \rightarrow(0, \infty)$ is said to be logarithmically convex in the Jensen sense if

$$
f\left(\frac{x+y}{2}\right) \leq \sqrt{f(x) f(y)}
$$

holds for all $x, y \in I$.

Definition 7 A function $f: I \rightarrow(0, \infty)$ is said to be logarithmically convex or log-convex if

$$
f((1-\lambda) s+\lambda t) \leq f(s)^{1-\lambda} f(t)^{\lambda}
$$

holds for all $s, t \in I, \lambda \in[0,1]$.

Remark 7 If a function is continuous and log-convex in the Jensen sense then it is also log-convex. We can also easily see that for positive functions exponential convexity implies log-convexity (consider Definition 4 for $n=2$ ).

The following two lemmas are equivalent to the definition of convexity (see [2], p.2).

Lemma 2 Let $f: I \rightarrow \mathbb{R}$ be a convex function. Then for any $x_{1}, x_{2}, x_{3} \in I$ such that $x_{1}<x_{2}<$ $x_{3}$ the following is valid:

$$
\left(x_{3}-x_{2}\right) f\left(x_{1}\right)+\left(x_{1}-x_{3}\right) f\left(x_{2}\right)+\left(x_{2}-x_{1}\right) f\left(x_{3}\right) \geq 0 .
$$

Lemma 3 Let $f: I \rightarrow \mathbb{R}$ be a convex function. Then for any $x_{1}, x_{2}, y_{1}, y_{2} \in I$ such that $x_{1} \leq y_{1}$, $x_{2} \leq y_{2}, x_{1} \neq x_{2}, y_{1} \neq y_{2}$ the following is valid:

$$
\frac{f\left(x_{2}\right)-f\left(x_{1}\right)}{x_{2}-x_{1}} \leq \frac{f\left(y_{2}\right)-f\left(y_{1}\right)}{y_{2}-y_{1}} .
$$

In order to obtain results regarding the exponential convexity, we define the families of functions as follows.

For every choice of $2 l+1$ mutually different points $z_{0}, z_{1}, \ldots, z_{2 l} \in[\alpha, \beta]$ we define

- $\mathcal{F}_{1}=\left\{f_{t}:[\alpha, \beta] \rightarrow \mathbb{R}: t \in I\right.$ and $t \mapsto\left[z_{0}, z_{1}, \ldots, z_{2 l} ; f_{t}\right]$ is $n$-exponentially convex in the Jensen sense on $I\}$;

- $\mathcal{F}_{2}=\left\{f_{t}:[\alpha, \beta] \rightarrow \mathbb{R}: t \in I\right.$ and $t \mapsto\left[z_{0}, z_{1}, \ldots, z_{2 l} ; f_{t}\right]$ is exponentially convex in the Jensen sense on $I\}$; 
- $\mathcal{F}_{3}=\left\{f_{t}:[\alpha, \beta] \rightarrow \mathbb{R}: t \in I\right.$ and $t \mapsto\left[z_{0}, z_{1}, \ldots, z_{2 l} ; f_{t}\right]$ is 2-exponentially convex in the Jensen sense on $I$.

Theorem 14 Let $A$ be the linear functional defined as in (4.1) associated with family $\mathcal{F}_{1}$. Then the following statements hold:

(i) The function $t \mapsto A\left(f_{t}\right)$ is $n$-exponentially convex in the Jensen sense on $I$.

(ii) If the function $t \mapsto A\left(f_{t}\right)$ is continuous on $I$, then it is n-exponentially convex on $I$.

Proof Our proof proceeds similarly to the proof of Theorem 4.1 in [12].

The following corollary is an easy consequence of the previous theorem.

Corollary 1 Let $A$ be the linear functional defined as in (4.1) associated with family $\mathcal{F}_{2}$. Then the following statements hold:

(i) The function $t \mapsto A\left(f_{t}\right)$ is exponentially convex in the Jensen sense on $I$.

(ii) If the function $t \mapsto A\left(f_{t}\right)$ is continuous on $I$, then it is exponentially convex on $I$.

Corollary 2 Let $A$ be the linear functional defined as in (4.1) associated with family $\mathcal{F}_{3}$. Then the following statements hold:

(i) If the function $t \mapsto A\left(f_{t}\right)$ is continuous on I, then it is 2-exponentially convex on I. If $t \mapsto A\left(f_{t}\right)$ is additionally positive, then it is also log-convex on I. Furthermore, for every choice $r, s, t \in I$, such that $r<s<t$, we have

$$
\left[A\left(f_{s}\right)\right]^{t-r} \leq\left[A\left(f_{r}\right)\right]^{t-s}\left[A\left(f_{r}\right)\right]^{s-r} .
$$

(ii) If the function $t \mapsto A\left(f_{t}\right)$ is positive and differentiable on $I$, then for all $r, s, u, v \in I$ such that $r \leq u, s \leq v$, we have

$$
M_{r, s}\left(A, \mathcal{F}_{3}\right) \leq M_{u, v}\left(A, \mathcal{F}_{3}\right)
$$

where

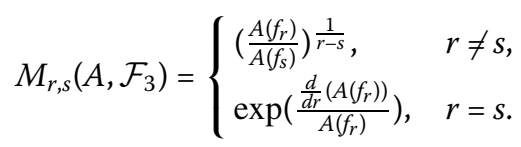

Proof (i) The first part of statement is an easy consequence of Theorem 14 and the second one of Remark 7.

Since the function $t \mapsto A\left(f_{t}\right)$ is $\log$-convex on $I$, i.e. the function $t \mapsto \log A\left(f_{t}\right)$ is convex on $I$, applying Lemma 2 we have

$$
(r-t) \log A\left(f_{t}\right)+(t-s) \log A\left(f_{r}\right)+(s-r) \log A\left(f_{r}\right) \geq 0
$$

for every choice $r, s, t \in I$, such that $r<s<t$. Therefore, we have

$$
\left[A\left(f_{s}\right)\right]^{t-r} \leq\left[A\left(f_{r}\right)\right]^{t-s}\left[A\left(f_{r}\right)\right]^{s-r}
$$


(ii) Applying Lemma 3 to the convex function $t \mapsto \log A\left(f_{t}\right)$, we get

$$
\frac{\log A\left(f_{r}\right)-\log A\left(f_{s}\right)}{r-s} \leq \frac{\log A\left(f_{u}\right)-\log A\left(f_{v}\right)}{u-v}
$$

for $r \leq u, s \leq v, r \neq u, s \neq v$. Therefore, we have

$$
M_{r, s}\left(A, \mathcal{F}_{3}\right) \leq M_{u, v}\left(A, \mathcal{F}_{3}\right) .
$$

The case $r=s, u=v$ follows from (4.4) as a limit case.

Remark 8 Note that, with the assumption that the functions from $\mathcal{F}_{1}, \mathcal{F}_{2}, \mathcal{F}_{3}$ are differentiable, the results from Theorem 14, Corollary 1, and Corollary 2 still hold when two of the points $z_{0}, z_{1}, \ldots, z_{2 l} \in[\alpha, \beta]$ coincide. Further, if the functions from $\mathcal{F}_{1}, \mathcal{F}_{2}, \mathcal{F}_{3}$ are $2 l$-times differentiable, the results still hold when all points coincide. These results can easily be proved using (1.10) and some facts as regards the exponential convexity.

\section{Applications to means}

Using some families of convex functions which are given below, we construct different examples of exponentially convex functions. As consequences, applying the mean-value theorem of Cauchy type from the previous section to these special families of functions, we establish new classes of two-parameter Cauchy type means that are symmetric and have monotone properties over both parameters.

Throughout this section id denotes the identity function, i.e. $\operatorname{id}(x)=x$ for each $x \in \mathbb{R}$.

Example 1 Consider the family of functions

$$
\Omega_{1}=\left\{\varphi_{t}:(0, \infty) \rightarrow \mathbb{R}: t \in \mathbb{R}\right\}
$$

defined by

$$
\varphi_{t}(x)= \begin{cases}\frac{x^{t}}{t(t-1) \cdots(t-2 n+1)}, & t \notin\{0,1, \ldots, 2 n-1\}, \\ \frac{x^{j} \log x}{(-1)^{2 n-1-j j !(2 n-1-j) !},} & t=j \in\{0,1, \ldots, 2 n-1\} .\end{cases}
$$

Since $\frac{d^{2 n} \varphi_{t}}{d x^{2 n}}(x)=x^{t-2 n}=e^{(t-2 n) \log x}>0, t \in \mathbb{R}$, it follows that $\varphi_{t}$ is $2 n$-convex on $(0, \infty)$ for every $t \in \mathbb{R}$ and $t \mapsto \frac{d^{2 n} \varphi_{t}}{d x^{2 n}}(x)$ is exponentially convex (for more explanations see [12]). Therefore, using the same arguments as in the proof of Theorem 14 we conclude that the function $t \mapsto\left[z_{0}, z_{1}, \ldots, z_{2 l} ; \varphi_{t}\right]$ is exponentially convex (and so exponentially convex in the Jensen sense). Then from Corollary 1 it follows that $t \mapsto A\left(\varphi_{t}\right)$ is exponentially convex in the Jensen sense. It is easy to verify that the function $t \mapsto A\left(\varphi_{t}\right)$ is continuous. Then from Corollary 1 it follows that $t \mapsto A\left(\varphi_{t}\right)$ is exponentially convex. In this case we assume that $[\alpha, \beta] \subset(0, \infty)$.

For this family of functions, with the assumption that $t \mapsto A\left(\varphi_{t}\right)$ is positive, (4.3) becomes

$$
M_{r, s}\left(A, \Omega_{1}\right)= \begin{cases}\left(\frac{A\left(\varphi_{r}\right)}{A\left(\varphi_{s}\right)}\right) \frac{1}{r-s}, & r \neq s, \\ \exp \left(-(2 n-1) ! \frac{A\left(\varphi_{r} \cdot \varphi_{0}\right)}{A\left(\varphi_{r}\right)}+\sum_{i=0}^{2 n-1} \frac{1}{i-r}\right), & r=s \notin\{0,1, \ldots, 2 n-1\}, \\ \exp \left(-(2 n-1) ! \frac{A\left(\varphi_{r} \cdot \varphi_{0}\right)}{2 A\left(\varphi_{r}\right)}+\sum_{i=0, i \neq r}^{2 n-1} \frac{1}{i-r}\right), & r=s \in\{0,1, \ldots, 2 n-1\} .\end{cases}
$$

Here we obtain extensions by continuity for a different choice of parameters $r, s \in \mathbb{R}$. 
Applying Theorem 13 to the functions $f=\varphi_{r}$ and $g=\varphi_{s}$, where $r, s \in \mathbb{R}, r \neq s, r, s \neq 0,1$ and setting $\alpha=\min _{i, j}\left\{x_{j}, y_{i}\right\}$ and $\beta=\max _{i, j}\left\{x_{j}, y_{i}\right\}$, it follows that there exists a unique $\xi \in$ $[\alpha, \beta]$ such that

$$
\xi^{r-s}=\frac{A\left(\varphi_{r}\right)}{A\left(\varphi_{s}\right)}
$$

i.e. since the function $\xi \mapsto \xi^{r-s}$ is invertible, we obtain

$$
\xi=\left(\frac{A\left(\varphi_{r}\right)}{A\left(\varphi_{s}\right)}\right)^{\frac{1}{r-s}}
$$

which represents a mean of the interval $[\alpha, \beta]$.

This shows that (5.1) represents the new class of two-parameter Cauchy type means which are obviously symmetric. Monotonicity over the two parameters $r$ and $s$ follows from part (ii) of Corollary 2.

Example 2 Consider the family of functions

$$
\Omega_{2}=\left\{\psi_{t}: \mathbb{R} \rightarrow[0, \infty): t \in \mathbb{R}\right\}
$$

defined by

$$
\psi_{t}(x)= \begin{cases}\frac{e^{t x}}{t^{2 n}}, & t \neq 0, \\ \frac{x^{2 n}}{(2 n) !}, & t=0 .\end{cases}
$$

Since $\frac{d^{2 n} \psi_{t}}{d x^{2 n}}(x)=e^{t x}>0, t \in \mathbb{R}$, it follows that $\psi_{t}$ is $2 n$-convex on $\mathbb{R}$ for every $t \in \mathbb{R}$ and $t \mapsto$ $\frac{d^{2 n} \psi_{t}}{d x^{2 n}}(x)=e^{t x}$ is exponentially convex by definition. Therefore, using the same arguments as in the previous example, we see that the function $t \mapsto A\left(\psi_{t}\right)$ is exponentially convex.

For this family of functions, with the assumption that $t \mapsto A\left(\psi_{t}\right)$ is positive, (4.3) becomes

$$
M_{r, s}\left(A, \Omega_{2}\right)= \begin{cases}\left(\frac{A\left(\psi_{r}\right)}{A\left(\psi_{s}\right)}\right) \frac{1}{r-s}, & r \neq s, \\ \exp \left(\frac{A\left(\mathrm{id} \cdot \psi_{r}\right)}{A\left(\psi_{r}\right)}-\frac{2 n}{r}\right), & r=s \neq 0, \\ \exp \left(\frac{1}{2 n+1} \frac{A\left(\mathrm{id} \cdot \psi_{0}\right)}{A\left(\psi_{0}\right)}\right), & r=s=0\end{cases}
$$

Applying Theorem 13 to this family of functions, it follows that

$$
\bar{M}_{r, s}\left(A, \Omega_{2}\right):=\log M_{r, s}\left(A, \Omega_{2}\right)
$$

represents the new class of two-parameter Cauchy type means of interval $[\alpha, \beta]$. The means from this class $\bar{M}_{r, s}\left(A, \Omega_{2}\right)$ are obviously symmetric. Monotonicity over both parameters follows from part (ii) of Corollary 2.

Example 3 Consider the family of functions

$$
\Omega_{3}=\left\{\lambda_{t}:(0, \infty) \rightarrow(0, \infty): t \in(0, \infty)\right\}
$$


defined by

$$
\lambda_{t}(x)= \begin{cases}\frac{t^{-x}}{(\log t)^{2 n}}, & t \neq 1, \\ \frac{x^{2 n}}{(2 n) !}, & t=1 .\end{cases}
$$

Since $t \mapsto \frac{d^{2 n} \lambda_{t}}{d x^{2 n}}(x)=t^{-x}>0, t \in(0, \infty)$, it follows that $\lambda_{t}$ is $2 n$-convex on $(0, \infty)$ for every $t \in(0, \infty)$ and $t \mapsto \frac{d^{2 n} \lambda_{t}}{d x^{2 n}}(x)=t^{-x}$ is exponentially convex since it is the Laplace transform of a nonnegative function (for more explanations see $[12,15]$ ). Therefore, using the same arguments as in the previous example, we see that the function $t \mapsto A\left(\lambda_{t}\right)$ is exponentially convex. In this case we assume that $[\alpha, \beta] \subset(0, \infty)$.

For this family of functions, with the assumption that $t \mapsto A\left(\varphi_{t}\right)$ is positive, (4.3) becomes

$$
M_{r, s}\left(A, \Omega_{3}\right)= \begin{cases}\left(\frac{A\left(\lambda_{r}\right)}{A\left(\lambda_{s}\right)}\right) \frac{1}{r-s}, & r \neq s, \\ \exp \left(-\frac{A\left(\mathrm{id} \cdot \lambda_{r}\right)}{r A\left(\lambda_{r}\right)}-\frac{2 n}{r \log r}\right), & r=s \neq 1, \\ \exp \left(-\frac{1}{2 n+1} \frac{A\left(\mathrm{id} \cdot \lambda_{1}\right)}{A\left(\lambda_{1}\right)}\right), & r=s=1 .\end{cases}
$$

For this family of functions, applying Theorem 13, we define the new class of twoparameter Cauchy type means as follows:

$$
\bar{M}_{r, s}\left(A, \Omega_{3}\right):=-L(r, s) \log M_{r, s}\left(A, \Omega_{3}\right) .
$$

Here $L(r, s)$ represents the logarithmic mean which is defined as follows:

$$
L(r, s)= \begin{cases}\frac{r-s}{\log r-\log s}, & r \neq s, \\ s, & r=s .\end{cases}
$$

The means from this class are obviously symmetric. Monotonicity with respect to the two parameters follows from part (ii) of Corollary 2.

Example 4 Consider the family of functions

$$
\Omega_{4}=\left\{\kappa_{t}:(0, \infty) \rightarrow(0, \infty): t \in(0, \infty)\right\}
$$

defined by

$$
\kappa_{t}(x)=\frac{e^{-x \sqrt{t}}}{t^{n}} .
$$

Since $t \mapsto \frac{d^{2 n} \kappa_{t}}{d x^{2 n}}(x)=e^{-x \sqrt{t}}, t \in(0, \infty)$, it follows that $\kappa_{t}$ is $2 n$-convex on $(0, \infty)$ for every $t \in$ $(0, \infty)$ and $t \mapsto \frac{d^{2 n} \kappa t}{d x^{2 n}}(x)=e^{-x \sqrt{t}}$ is exponentially convex since it is the Laplace transform of a nonnegative function (for more details see $[12,15]$ ). Therefore, using the same arguments as in the previous example, we see that the function $t \mapsto A\left(\kappa_{t}\right)$ is exponentially convex. In this case we assume that $[\alpha, \beta] \subset(0, \infty)$.

For this family of functions, with the assumption that $t \mapsto A\left(\varphi_{t}\right)$ is positive, (4.3) becomes

$$
M_{r, s}\left(A, \Omega_{4}\right)= \begin{cases}\left(\frac{A\left(\kappa_{r}\right)}{A\left(\kappa_{s}\right)}\right) \frac{1}{r-s}, & r \neq s, \\ \exp \left(-\frac{A\left(\mathrm{~d} \cdot \kappa_{r}\right)}{2 \sqrt{r} A\left(\kappa_{r}\right)}-\frac{n}{r}\right), & r=s .\end{cases}
$$


For this family of functions, applying Theorem 13, we define the new class of twoparameter Cauchy type means as follows:

$$
\bar{M}_{r, s}\left(A, \Omega_{4}\right):=-(\sqrt{r}+\sqrt{s}) \log M_{r, s}\left(A, \Omega_{3}\right)
$$

and the means from this class are obviously symmetric. Monotonicity with respect to the two parameters follows from part (ii) of Corollary 2.

\section{Conclusions}

In this paper we give generalizations of Sherman's theorem from which a majorization theorem follows as a special case. Our results hold for real, not necessarily nonnegative entries of vectors $\mathbf{a}, \mathbf{b}$ and matrix $\mathbf{A}$, as is the case of Sherman's theorem, and for $2 n$-convex functions which are in a special case convex in the usual sense. The methods used are based on classical real analysis and the application of Lidstone's interpolating polynomials and exponential convexity method and can be extended to the investigation of other inequalities.

\section{Competing interests}

The authors declare that they have no competing interests.

\section{Authors' contributions}

All authors contributed equally to deriving all the results of this article, and read and approved the final manuscript.

\section{Author details}

1 Department of Mathematics, Texas A\&M, University-Kingsville, Rhode Hall 217B, MSC 172, Kingsville, TX 78363-8202, USA. ${ }^{2}$ Faculty of Civil Engineering, Architecture and Geodesy, University of Split, Matice hrvatske 15, Split, 21000, Croatia.

${ }^{3}$ Faculty of Textile Technology, University of Zagreb, Prilaz Baruna Filipovića 30, Zagreb, 10000, Croatia.

Received: 15 September 2015 Accepted: 6 December 2015 Published online: 04 January 2016

\section{References}

1. Hardy, GH, Littlewood, JE, Pólya, G: Inequalities, 2nd edn. Cambridge University Press, Cambridge (1952)

2. Pečarić, JE, Proschan, F, Tong, YL: Convex Functions, Partial Orderings, and Statistical Applications. Academic Press, Boston (1992)

3. Fuchs, L: A new proof of an inequality of Hardy, Littlewood and Pólya. Mat. Tidsskr., B 1947, $53-54$ (1947)

4. Niezgoda, M: Remarks on Sherman like inequalities for $(\alpha, \beta)$-convex functions. Math. Inequal. Appl. 17(4), 1579-1590 (2014)

5. Sherman, S: On a theorem of Hardy, Littlewood, Pólya and Blackwell. Proc. Natl. Acad. Sci. USA 37(1), 826-831 (1957)

6. Lidstone, GJ: Notes on the extension of Aitken's theorem (for polynomial interpolation) to the Everett types. Proc. Edinb. Math. Soc. (2) 2, 16-19 (1929)

7. Boas, RP: Representation of functions by Lidstone series. Duke Math. J. 10, 239-245 (1943)

8. Poritsky, H: On certain polynomial and other approximations to analytic functions. Trans. Am. Math. Soc. 34, 274-331 (1932)

9. Widder, DV: Completely convex function and Lidstone series. Trans. Am. Math. Soc. 51, 387-398 (1942)

10. Agarwal, RP, Wong, PJY: Error Inequalities in Polynomial Interpolation and Their Applications. Kluwer Academic, Dordrecht (1993)

11. Cerone, P, Dragomir, SS: Some new Ostrowski-type bounds for the Čebyšev functional and applications. J. Math. Inequal. 8(1), 159-170 (2014)

12. Jakšetić, J, Pečarić, J: Exponential convexity method. J. Convex Anal. 20(1), 181-197 (2013)

13. Khan, AR, Pečarić, J, Rodić Lipanović, M: n-Exponential convexity for Jensen-type inequalities. J. Math. Inequal. 7(3), 313-335 (2013)

14. Pečarić, J, Perić, J: Improvement of the Giaccardi and the Petrović inequality and related Stolarsky type means. An. Univ. Craiova, Ser. Mat. Inform. 39(1), 65-75 (2012)

15. Schiff, JL: The Laplace Transform: Theory and Applications. Undergraduate Texts in Mathematics. Springer, New York (1999) 